\title{
Protée
}

\section{De la signalétique à l'emblématique : Twining de Charles Loupot}

\section{Pierre Fresnault-Deruelle}

Volume 35, numéro 1, printemps 2007

Échos et résonances

URI : https://id.erudit.org/iderudit/015892ar

DOI : https://doi.org/10.7202/015892ar

Aller au sommaire du numéro

Éditeur(s)

Département des arts et lettres - Université du Québec à Chicoutimi

ISSN

0300-3523 (imprimé)

1708-2307 (numérique)

Découvrir la revue

Citer cet article

Fresnault-Deruelle, P. (2007). De la signalétique à l'emblématique : Twining de Charles Loupot. Protée, 35(1), 87-92. https://doi.org/10.7202/015892ar
Résumé de l'article

Ce texte essaie d'explorer quelques-unes des relations entre le texte et l'image dans les placards publicitaires des années 1930. L'auteur tente également de dégager en quoi ressurgit une certaine économie de l'emblème qu'il associe à ce qu'il pense être la pensée visuelle. Avec cette courte étude, quelques éléments sont fournis sur ce qu'il en serait d'une esthétique de la communication. d'utilisation que vous pouvez consulter en ligne.

https://apropos.erudit.org/fr/usagers/politique-dutilisation/ 


\title{
DE LA SIGNALÉTIQUE À L'EMBLÉMATIQUE TWINING DE CHARLES LOUPOT
}

\author{
Pierre Fresnault-DeruelLe
}

\section{«L'ÉPAISSEUR» DES IMAGES}

Une image donnée n'est jamais que la énième version $d^{\prime}$ un iconogramme auquel on la peut rattacher. C'est toujours, en vérité, une image d'image. Cela signifie qu'une image n'a de «vertu évocante» qu'à proportion de sa «vertu révocante». Précisons qu'exception faite du cas particulier constitué par les représentations « citationnelles» ou parodiques, une image fonctionne d'autant mieux qu'elle refoule d'autres images - sœurs ou cousines - qui I'informent en secret. Or, c'est ce secret-là qu'il nous faut lever si nous voulons lire tel portrait, tel dessin d'humour, telle photographie de presse, etc., étant entendu que ce qu'on appelle « lecture » suppose de la part du lecteur, outre une capacité combinatoire, une certaine compétence systémique (ou paradigmatique) - bref, une certaine «conscience intertextuelle ${ }^{1}$. En somme, et pour originale qu'elle soit, une image est toujours à la croisée de formes migrantes, stabilisées à l'occasion par un peintre, un graphiste ou un photographe. Offerte au lecteur, I'image donne donc à ce dernier l'opportunité d'apprécier l'esprit d'à-propos qu'elle recèle, c'est-à-dire la façon dont elle prend sa place dans le texte de la culture.

\section{LA LETTRE ARCHITECTURÉE}

Ces considérations faites, fixons-nous sur I'affiche fameuse de Loupot intitulée Twining (ill. 1)

Cette affiche se retrouve dans toutes les anthologies consacrées aux grands affichistes. Essayons d'expliciter les raisons qui font de ce placard un artefact exemplaire.

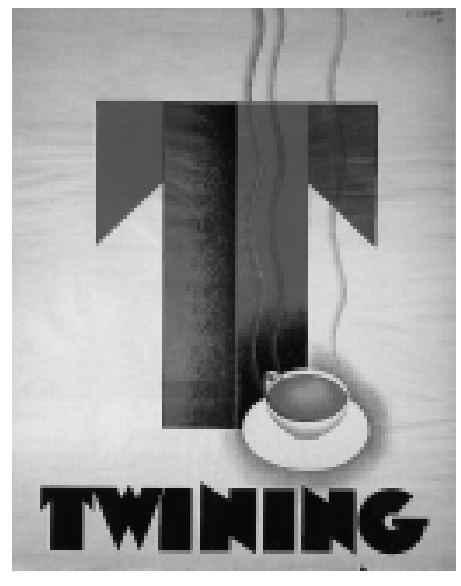

Illustration 1

Charles Loupot, Twining, 1930, lithogravure, $160 \times 120 \mathrm{~cm}$. (C) Succession Charles Loupot / SODRAC 2007

On sait que les recherches en typographie suscitent depuis des siècles nombre de traités où les lettres, architecturées, sont accompagnées de protocoles de construction, parfois fort savants. Si le lettrage de la réclame Twining, empiriquement établi, ne ressortit pas aux strictes règles de l'art (sauf erreur, ce lettrage n'est extrait d'aucun alphabet déposé), force est d'admettre, toutefois, que les caractères de cette réclame, en particulier le grand $\mathrm{T}$, font de cette affiche-objet (I'objet réduit à son signe) une image édifiante à tous les sens du terme.

La «différence de potentiel » instaurée par Loupot entre l'initiale majuscule du mot «Twining " et le dessin de la tasse signifie que quelque chose est expressément outre- 
passé dans l'ordre de la représentation, de sorte que nous éprouvons, fût-ce confusément, le sentiment du gigantesque. Paradoxalement, cet effet est d'autant mieux ressenti que I'affiche est de taille moyenne, ce qui signifie que ledit effet tient plus de l'espace symbolique du placard que de sa surface réelle. Le principe au travail ici est le même mais inversé - qui voudra que le bébé Benetton expose, dans les années 1980, son «énorme petitesse » sur les douze mètres carrés d'une affiche format métro. Bref, c'est avec un métier consommé que Loupot promeut l'idée même de promotion, qui commande que ses images soient d'abord des magnifications au sens premier du terme: I'affiche Twining est par excellence sémaphorique.

Toute affiche réussie, autant qu'un manifeste convaincant chargé de vanter un objet, un service ou une politique, est au premier chef une démonstration en acte de la vertu du support utilisé. De fait, Twining célèbre d'abord la force rhétorique du dessin de prise en charge. Nous pensons, à cet égard, qu'une performance langagière un tant soit peu élaborée (formule poétique, trouvaille graphique, etc.) nous entretient quasi nécessairement de «l'intelligence sémiotique» qui l'a permise. Notons, encore, que le T est une forme «surdéterminée » en matière graphique. On veut dire que sa version romaine est l'orthogonalité même et, qu'avec elle, nous avons les dimensions du gabarit rectangulaire où cette lettre est censée s'inscrire. À l'instar du symbole chrétien, centré, sujet de maints retables, le T est au principe même de la surface qu'il comprend et qui le comprend - mutatis mutandis, en 1970, Jean Widmer réinvente le $\mathrm{T}$ pour saluer une des premières expositions du Centre de Création Industrielle à Paris intitulée «à Table».

\section{DESCRIPTION, ANALYSE, COMPARAISONS STYLISTIQUES}

Au pied de la lettre géante (rouge et brune), une tasse de thé, vue en plongée, se donne à la fois comme objet disposé et comme offrande déposée. L'ordinaire profane se mâtine de «ritualité ». Se voit donc ici associée au signe du réconfort la marque d'un cérémonial. Cela a pour effet $d^{\prime}$ inviter le lecteur/spectateur à se compter au rang des sectateurs de la high life qui voulait, en 1930, qu'on fût anglophile, partant, qu'on considérât le café comme une boisson trop ordinaire. En ce temps-là, Twining, c'était un

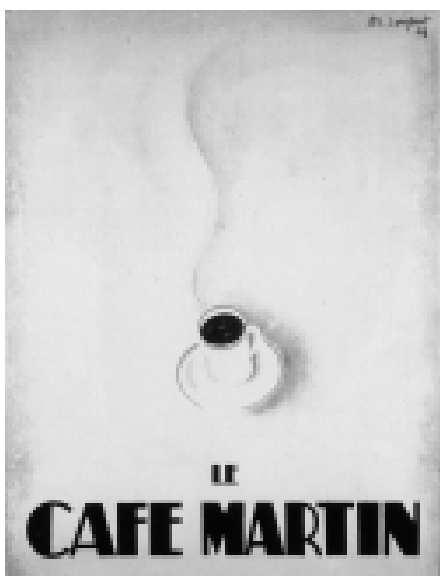

Illustration 2

Charles Loupot, Le Café Martin, 1929. (C) Succession Charles Loupot / SODRAC 2007

peu de la britannicité chic importée en France. Pourtant, c'est d'une publicité pour le café - en vérité, sobre et fort élégante - que procède I'affiche Twining. En effet, un an auparavant (1929), Loupot conçoit un placard pour le Café Martin (ill. 2) qui recourt au même signe graphique - chargé de représenter l'olfaction - utilisé pour l'exaltation du thé.

Avec l'affiche Twining, Loupot reprend donc la sinusoïdale du café en question au motif précisément qu'elle fait partie du «vocabulaire» plastique des Arts déco (voir infra). Ajoutons que le rouge orangé du liquide contenu dans la tasse, ton repris en majeur par la partie droite du $T$, renforce d'autant "l'ascendance» de l'arôme dégagé par ledit thé. On se plaît à imaginer que la vapeur, atteignant le haut de l'affiche, déborde du cadre. Aussi, après avoir infusé, Twining se diffuse-t-il, remplissant l'espace (le nôtre) de sa senteur raffinée. Les volutes qui s'échappent du précieux liquide animent avec grâce la composition. L'époque veut que, des rigueurs géométriques de tel ou tel ensemble ou motif donné, des courbes - certes contraintes viennent adoucir la possible sécheresse.

Évoquons cette tête de femme dessinée, sur un paravent $(1931)^{2}$, par l'Américain Donald Deskey, chez qui se devine l'influence du purisme de Le Corbusier; cette réclame de Jean Carlu (ill. 3), conçue pour I'aquarium de Monaco, placard sur lequel une ondulante murène vient offusquer le poisson plat qui lui donne son assiette; ou bien encore l'ad- 


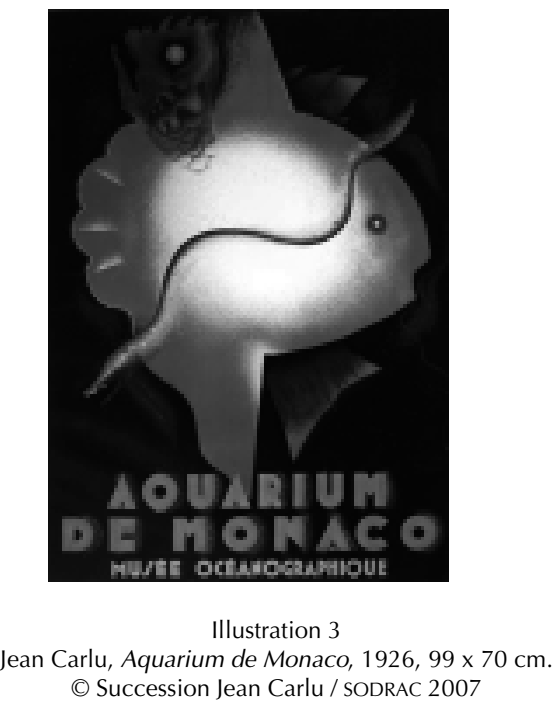

mirable publicité pour les cigarettes Modiano de Berény (1927) ${ }^{3}$ où le géométrisme et l'obliquité du dessin se voient « corrigés » par la paradoxale orthogonalité de deux lignes serpentines, dénotant respectivement la consumation du tabac et le rejet, par le fumeur, de la fumée inhalée.

\section{DE LA LETTRE HISTORIÉE À L'IDÉOGRAMME}

Ce T géant, aux ailes déployées (on dirait également un totem indien), qui domine de sa masse le mot "Twining», est à la fois l'initiale du nom et l'emblème de la marque. Concernant le principe sémiotique qui a présidé à l'élaboration de ce manifeste, il est évident que Loupot a voulu que le T (té) symbolise ici le thé (Twining). Ce type de figures, remarquable chez les grands affichistes (Cassandre, Colin, Savignac, etc.), est à notre avis une manifestation de ce qu'on nomme la "pensée visuelle», concept flou dont on perçoit, cependant, qu'il a trait à la manière dont l'image court-circuite la linéarité verbale ${ }^{4}$. On fait ici le pari que Loupot, en élaborant son affiche, fut plus à l'écoute de son intuition que de la raison analytique. Quoi qu'il en soit, Loupot, en dessinant ce qui se présente comme un rébus, renoue avec l'idéogrammatisme, dont on sait qu'il fut longtemps confiné sous le boisseau ${ }^{5}$.

Sans doute, un autre exemple de ce qu'est, pour nous, la pensée visuelle ne serait-il pas de trop. Soit ce placard de Cappiello destiné à exalter les mérites du Chocolat Klaus (1903).

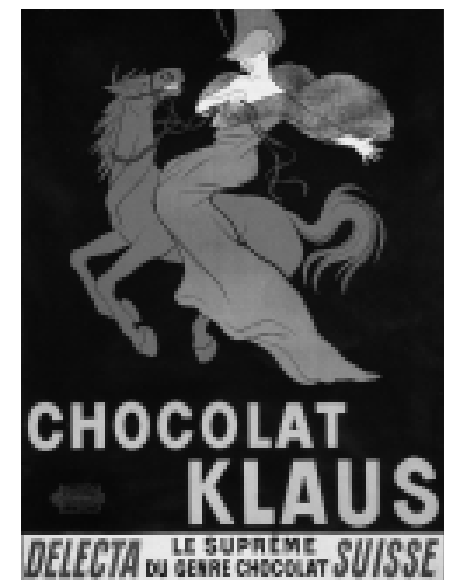

Illustration 4

Leonetto Cappiello, Chocolat Klaus, 1903, $160 \times 118 \mathrm{~cm}$ (C) Succession Leonetto Cappiello / SODRAC 2007

Une belle fille tout de vert vêtue est assise, en amazone, sur un fougueux destrier rouge. Cette image, qui prend sa place dans la longue lignée des représentations de La Belle et La Bête, illustre une nouvelle fois le thème de l'efficience du charme féminin. Mais, quel rapport, dira-t-on, $y$-a t-il entre ce que nous voyons et ce que nous lisons? La réponse à cette question prend corps si nous comprenons que le déphasage de l'image par rapport au texte peut être dépassé; en un mot, si nous saisissons que, du cheval à I'amazone, se tisse un lien analogue à celui censé exister entre le Chocolat Klaus et le public qui ne demande qu'à saliver. Bref, sur ce fond d'écran noir (cet « écran » des fantasmes), la «constellation» rouge et vert de Cappiello se présente comme le chiffre de l'irrésistible. Le cheval est à sa maîtresse ce que Klaus est au désir des gourmands. Le désarmant secret de cette affiche vient de ce que notre hypothèse interprétative n'est pas directement saisissable sur I'affiche qui, cependant, subsume visuellement ces pensées 6 , perçues à «mi-mots ».

Revenons à Loupot et aux affichistes de son entourage. Le logocentrisme n'a pas, bien entendu, dit son dernier mot. Peu de temps après Twining, l'affichiste Paul Colin propose, en 1933 (ill. 5), l'affiche Prenez $L$ 'R, pour l'apéritif «R», évidemment baptisée de la sorte après qu'il ait imaginé l'amphibologie: «Prenez l'air » / «Prenez I'R ». L'affiche de Colin représente un $\mathrm{R}$ énorme, rouge, dont le cen- 


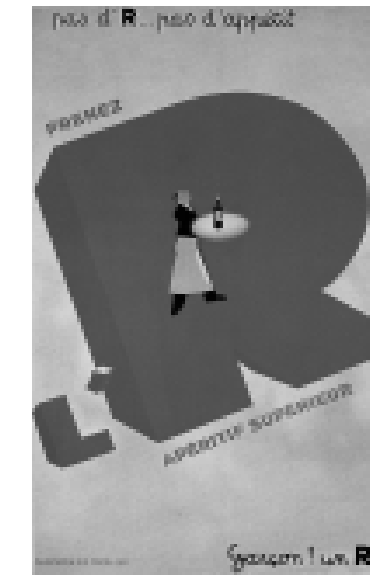

Illustration 5

Paul Colin, Prenez L'R, 1933, $150 \times 99 \mathrm{~cm}$.

(C) Succession Paul Colin / SODRAC 2007

tre, évidé, est occupé par un garçon de café sur le plateau duquel se trouve une bouteille de la marque de référence. Or, parce qu'il est un rien suffisant, parce qu'il ne «manque pas d'air» (comprenez aussi « $\left.d^{\prime} R »\right)$, le garçon, qui répond à la commande (Garçon ! un $R$ ), est-il à même d'affecter I'arrogance (I'air supérieur) de qui se sent dépositaire d'un objet convoité (l'apéritif «R»).

Nous observons que, si I'affiche de Colin est apparemment bien plus astucieuse que celle de Loupot, la prégnance plastique et signalétique de Twining l'emporte, in fine, en opérativité. Le T de Loupot ressortit en effet à la signalétique, tandis que le R de Colin - plus « intellectuel» - aurait mieux convenu à un support plus modeste (une annonce dans un journal, une carte postale publicitaire ). Bref, I'amusante rhétorique de Prenez $L^{\prime} R$ (= quittez vos habitudes) aurait été sans doute plus appropriée à un petit format qu'à un placard destiné à attirer l'œil du passant pressé. S'il ne convient pas de «se prendre la tête» pour considérer à sa juste valeur le placard de Colin, force est d'admettre toutefois que nous tenons là un «texte» dont l'économie visuelle est impropre à l'attention «flottante» requise par Loupot ou Cappiello.

Cela nous amène au lien qui unit les images visuelles de nos publicitaires à l'imagerie verbale dont elles dépendent. C'est, en effet, avec l'engramme d'expressions toutes faites, de mots ou de lettres-rébus avec lesquelles elles font système que certaines icônes doivent être interrogées. Le T

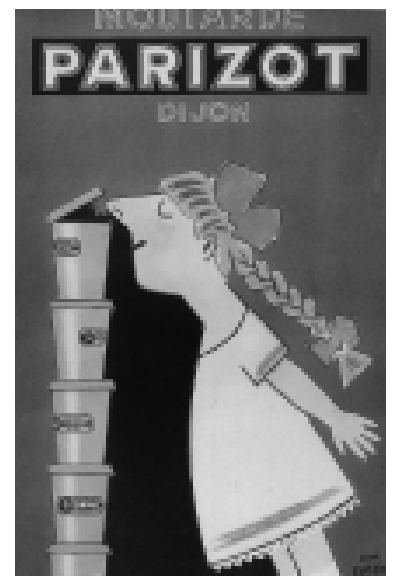

Illustration 6

Raymond Savignac, Moutarde Parizot, 1952.

(C) Succession Raymond Savignac / SODRAC 2007

de Loupot ou le $\mathrm{R}$ de Colin «résonnent» en nous pour la raison que ces graphèmes activent le souvenir du son des lettres ou de certains mots (sub)vocalisés.

Faut-il penser que le lien entre l'image et la lettre écrite, voire le sous-texte illustré, procède d'une dialectique psychique (préconsciente) qui s'apparenterait à ce qu'Edgar Morin nomme «dialogique ${ }^{7}$ ? Les ordres symboliques du linguistique (linéaire et analytique) et de l'iconique, (tabulaire et synthétique) qui semblent se conforter, ici, tout en s'opposant, nous invitent à le croire.

Voyez, encore, à cet égard, cette affiche de Savignac (ill. 6) chargée de nous entretenir des mérites de la moutarde Parizot. Curieusement, la fillette, dont le nez s'est allongé pour être à la bonne hauteur du dernier pot de la pile de verres, semble ravie de humer le «parfum » du condiment. Est-ce forcer les choses de dire que la marque Parizot est d'autant plus valorisée que la « démonstration» repose sur une sorte d'antilogie surmontée? Comment ne pas remarquer, en effet, que le dessin de Savignac est la version positive du cliché linguistique «la moutarde qui monte au nez », la colère ayant fait place à la délectation? Chose étonnante, l'expérience (la réponse à la question posée autour de soi) prouve que cette affiche n'est pas décodée comme telle, ce qui signifie que sa réception est tributaire $d^{\prime}$ une représentation mentale non consciemment «reformatée». Nous croyons en outre que, passé un certain seuil de perception, une proposition donnée et sa dé- 
négation sont interchangeables. En somme, et si tel est bien le cas, le plaisir de la lecture viendrait du fait que le dessin a pris « dialogiquement» en charge l'image mentale de départ, à savoir la métaphore de la colère naissante ( « la moutarde qui vous monte au nez »).

\section{RETOUR À L'AFFICHE DE LOUPOT:}

\section{UNE ESTHÉTIQUE DE LA COMMUNICATION}

Et la question de I'articulation du lisible au visible de ressurgir. Si le vu est à la compréhension ce que le lu est à I'extension, et si, par ailleurs, lire, c'est effectivement discriminer puis recombiner des unités en une synthèse reconstruite, il ressort que Twining, à l'opposite de Prenez $L^{\prime} R$, a la force d'un logotype. À preuve, Twining peut changer d'échelle de représentation sans déperdition symbolique majeure.

Twining, qui n'est, décidément, pas n'importe quelle marque, impose résolument son design. Elle est à la fois une image de marque et une enseigne. Récusant le pittoresque auquel on pouvait s'attendre, la marque arbore ce rien de simplicité aristocratique que lui confère ce $\mathrm{T}$ hiéroglyphique qui, du fait de I'homonymie ( $T$, té, thé) dont il est l'objet et le support, n'a pas cru devoir s'encombrer de signes redondants. Ainsi, le T de Twining - altier et multivalent - se suffit à lui-même! Cette affiche, à la simplicité savante, aurait pu déplaire, à tout le moins se fondre dans la production d'alors. Pourtant, il n'en est rien. Elle a séduit, ainsi qu'en témoignent la plupart des études consacrées à l'histoire des affiches. Pourquoi? Parce que Loupot fait la démonstration de ce que peut être une esthétique de la communication, à savoir cette "sémaphorique», rêvée par tous les grands affichistes ${ }^{8}$, où le graphisme, adapté à son propos, est la résultante $d^{\prime}$ une nécessité chaque fois réinventée. Marie-José Mondzain, dans un texte lumineux ${ }^{9}$, parle de ces publicitaires d'antan chez qui la nécessité de communiquer ne ravalait nullement le talent au rang d'un simple savoir-faire (si pratique pour évacuer ces productions «impures» de la sphère artistique). Cela confirme, soit dit en passant, que, s'il y a des principes à respecter, en communication visuelle comme ailleurs, il ne peut y avoir de recettes préétablies et, partant, nulle «grammaire» à appliquer.

\section{L'EMBLÉMATIQUE}

Il est connu qu'en matière de langage, la fonction dite «poétique» se manifeste par l'accent porté (plus ou moins fortement) sur le message. La forme dudit message est, au final, indissociable du propos tenu. Loupot, qui s'est spécialisé dans cette discipline qu'est l'affiche, réussit, avec Twining, à produire un emblème, c'est-à-dire ce signe grâce à quoi l'esprit d'une marque (autrefois: l'esprit d'une entité allégorique) condense "poétiquement » les qualités dont celle-ci prétend être détentrice. Tentons d'être plus précis et revenons une dernière fois au dispositif de notre affiche sur laquelle - on l'a remarqué d'entrée - le produit visé par la publicité est représenté en contrepoint du T surdimensionné. Très exactement, au pied de la lettre. Rappelons que «le pied de la lettre», ou littéralisation, est aussi le fait de ramener telle ou telle image verbale à son niveau sémantique premier, de sorte qu'il est soudain possible de dégager l'étrangeté qui la caractérise. Par exemple, l'expression «marcher sur des œufs», en lieu et place d'un message ordinaire disant qu'il convient de "parler avec grande précaution», peut être à l'origine d'un dessin des plus cocasses (cette littéralisation, objectivée au plan graphique, est un ressort de I'humour contemporain). Considéré de la sorte, "le pied de la lettre» se présente comme le lieu d'origine à partir duquel "déplacements» et autres " condensations » ne demandent qu'à se développer. Or, il se trouve que le pied de la lettre est pris «au pied de la lettre», comme s'il était possible, désormais, de savourer ce thé qui ne manque pas de grandeur!

\section{UNE ESTHÉTIQUE DE LA COMMUNICATION}

Élargissons le propos: en quoi Loupot atteint-il à cette esthétique fonctionnelle (ou appliquée) qu'on nomme « esthétique de la communication»? Dans la mesure, justement, où s'équilibrent autant l'économie sémiotique du message que l'appréciation même de cette économie, autrement dit, autant la mise en "conformité » de la cohérence des motifs tendus vers la transmission du message que la cohésion plastique qui les fédère. Vif est le plaisir éprouvé de saisir (même fugitivement) la capacité qu'a eue l'affichiste de faire de simplicité vertu, de ramener à une formule lisible un groupe restreint de signifiants réunis en vue de générer, au-delà de la signification escomptée, un peu 
de sens ajouté: Twining n'est pas un cadeau qu'on vous fait, c'est un don, une offrande (l'hyperbole n'étant suggérée qu'à partir de son inverse, l'euphémisme lapidaire).

Ce manifeste marque un tournant dans la production de Loupot (pour ne rien dire de l'histoire de l'affiche). Après ses premières armes en Suisse, l'artiste s'est éloigné des afféteries de l'Art Nouveau et du goût pour l'anecdote, au bénéfice d'un art «prompt», aux arêtes vives, pour lequel l'abréviation est devenue autant une nécessité qu'une exigence.

\section{NOTES}

1. L'intertexte (ensemble non clos) est le lieu des réverbérations entre une forme donnée (textuelle ou iconique) et les autres formes, in absentia, qui font système avec ladite forme donnée. La parodie et le pastiche sont des manifestations canoniques de l'intertexte. On ajoutera à ces dernières tout ce qui - précédent ou conséquent - fait d'un texte ou d'une image un avatar rattachable à d'autres textes ou d'autres images. De sorte qu'une image (ou un texte) un tant soit peu élaborée se trouve être le carrefour de plusieurs lignées de paradigmes.

2. En ligne: http://www.moderneage.com/screens.html (page consultée le 16 janvier 2007)

3. En ligne: http://www3.flickr.com/photos/lamarde/46360767/ (page consultée le 16 janvier 2007).

4. Nous sommes proches, ici, de la topique freudienne, à un niveau conscient-préconscient, celui des processus secondaires, où les représentations sont stabilisées.

5. Sur ce point, on se reportera au livre d'A.-M. Christin, L'Image écrite, Paris, Flammarion, 1995, p. 153 et suiv. ("La lettre à l'affiche»).

6 . La tentation est grande de retrouver ici quelque chose du rapport que Freud perçoit entre le rêve et les pensées du rêve.

7. On se réfère ici au texte $d^{\prime} E$. Morin reproduit sur Internet dans le Bulletin interactif du Centre international de recherches et études transdisciplinaires (CIRET), no 12, février 1998. En ligne: http://nicol.club.fr/ ciret/bulletin/b12/b12.htm] (page consultée le 16 janvier 2007). Texte repris dans La Méthode, V, Paris, Seuil, 2001.

8. Cassandre, lui-même, théorise sur ce point, servi il est vrai par une pratique dont tout un chacun ne pouvait se réclamer. Voir H. Mouron, Cassandre, Genève, Skira, 1985, p. 48-52.

9. Voir le catalogue du Festival de Chaumont, Chaumont 2004, Paris, Éd. Pyramyd, 2004. 